\title{
Editorial
}

\section{Applications of Air Trajectories}

\author{
Isidro A. Pérez, ${ }^{1}$ Florinda Artuso, ${ }^{2}$ Mastura Mahmud, ${ }^{3}$ Umesh Kulshrestha, ${ }^{4}$ \\ M. Luisa Sánchez, ${ }^{1}$ and M. Ángeles García ${ }^{1}$ \\ ${ }^{1}$ Department of Applied Physics, University of Valladolid, 47011 Valladolid, Spain \\ ${ }^{2}$ Diagnostics and Metrology Laboratory, ENEA, Frascati, 00044 Rome, Italy \\ ${ }^{3}$ School of Social, Development and Environment, Universiti Kebangsaan Malaysia, Bangi, Selangor 43600, Malaysia \\ ${ }^{4}$ School of Environmental Sciences, Jawaharlal Nehru University, New Delhi 110067, India
}

Correspondence should be addressed to Isidro A. Pérez; iaperez@fal.uva.es

Received 21 July 2014; Accepted 21 July 2014

Copyright (c) 2015 Isidro A. Pérez et al. This is an open access article distributed under the Creative Commons Attribution License, which permits unrestricted use, distribution, and reproduction in any medium, provided the original work is properly cited.

The impact of substances released into the atmosphere is observed on materials or living beings. However, the origin of air masses affecting receptors determines their initial features and transport from sources plays an important role, since injections during mass travelling or mixing with surrounding air parcels may cause significant changes in the original properties. Calculating air trajectories and subsequent analysis is required to obtain the best identification of places of origin, changes undergone by the travelling air parcel, and its possible effects on receptors.

Air trajectories basically measure the dynamical processes in the atmosphere, where the path of air mass motion is trailed at a specified location in space at a particular arrival time either as forward, indicating the imminent path taken by the particles, or backward, where the historical path of the particles had travelled along their trajectory. Wind and meteorological conditions play an important part in the calculations of the air trajectories.

This special issue focusing on applications of air trajectories is justified for a number of reasons. One is that this technique enables possible temporal and spatial analyses in order to obtain airflow patterns. Another interesting feature of this topic is its interdisciplinary nature, since air mass transport is linked to an extremely wide range of applications covering meteorological uses such as cyclone evolution, water vapour transport or precipitation, air pollution impact and source identification, pollution transport to remote sites, influence of dangerous substances, and effects on health or the scarcely explored transport of microorganisms, which might settle in regions outside their original environment. Another noticeable property is that research in this field reaches beyond conventional atmospheric studies centred on experimental description and analysis, since results of trajectory calculations, though interesting themselves, should be combined with measurements at the study site. In fact, this topic falls within the applied research that establishes a direct link between the theoretical calculations from meteorological measurements and other observed variables.

The main benefits of this research are a better knowledge of atmospheric processes, such as cloud formation and their evolution, and the possibility of minimizing the adverse effects of human activity on the environment, like air pollution transport, and reducing the costs that the effects of natural or anthropogenic sources have on human activity, such as respiratory diseases due to transport of particles or hazardous substances. In this sense, air trajectories are obtained from observations that cannot be changed whereas some of the variables transported, such as chemical concentrations, may be modified by applying control strategies if sources have been clearly identified. However, this control may not always be possible since sources sometimes originate in countries other than those where effects are observed or the implementation of control measures may prove extremely costly or even impossible. In this situation, the procedure concludes once the source that has been identified. On other occasions, natural sources, such as grasslands, forests, or deserts, cannot be modified and efforts aimed at reducing effects should focus on receptors. 
Air trajectories may be determined by a range of procedures such as web based tools, which are used worldwide. These programs produce numerical outputs that can be processed and plots that can be interpreted to provide visual representation. They are simple since only transport is considered and more complex transformations experienced by air masses are excluded. The only cost is the staff required to run the programs and the time used following the number of simulations. Additionally, different scenarios may be proposed to observe the model response. Calculating large numbers of trajectories involves subsequent statistical treatment to obtain results by assimilation processes, such as cluster analysis and calculating the potential source contribution function. In general, spatial analysis techniques can be implemented. However, a low number of trajectories is normally used for ancillary or merely descriptive purposes. At present, few new models are being developed, with research focusing on the intensive application of existing models. Although these have been extensively checked, validation is still required to improve their performance.

Trajectory analysis is sometimes an irreplaceable tool. Illustrative examples are distinguishing between local influences and long-range transport, since all spatial scales may be covered, or determining orographic influences on the airflow, such as valleys or ridges. Moreover, atmospheric circulation at a planetary scale may be successfully observed, in particular, exchange between seas and continents, which influences the moisture content of the air mass and the transition between low and high latitudes, which implies temperature changes or between low and high altitudes, where the air composition is different. Determining air trajectory is sometimes recommendable, as in the case of an adverse event that can affect densely populated sites. Another interesting application is observing the contrast in air mass behaviour at different heights due to the wind shear. One noticeable feature of the trajectory is its shape, since straight trajectories over the same type of surface indicate a direct relationship between departure and arrival sites. However, meandering trajectories may reveal changing winds with intensive mixing processes, for instance, due to the influence of pressure systems and loss of initial characteristics.

Although this topic may seem uncommon, atmospheric research frequently uses air trajectory determination. However, this approach is normally secondary, since research papers usually focus on measurements. The main reasons for the absence of such studies in papers may be the belief that trajectory calculations are felt to be complicated or because of unfamiliarity with models. However, a look at the applications shows that this is an evolving field where calculation techniques have developed intensively and where applications are frequent but in some way remain isolated. The relative isolation of this research, far from being a disadvantage, opens the way for further studies to establish air flow patterns at higher scales. Another feature of this evolving field is that multiple variables are involved, which sometimes makes it difficult to decide which variable is being emphasised.

This special issue is therefore necessary to highlight a topic which has been increasingly used in recent years.
This positive trend indicates that results respond to the expectations placed in this technique, since precise knowledge of the path followed by an air mass is necessary to determine the impact of sources on receptors together with the concentration measurement, which is a salient although limited part. Additionally, the simplicity and wide scope of applications points to a growing future use and encourages research aimed at gaining insights into the achievements, limitations, and uncertainties that provide feedback and can improve air trajectory determination.

This issue is conceived to gather the current state of knowledge in this field by research that involves specific applications of air trajectories. The result is a mosaic of research areas and geographical areas where this topic is active, thus showing which avenues may be explored at the present time.

The interest of this special issue is twofold. Firstly, applications normally published in dispersed journals are brought together and secondly, the references reviewed cover the bulk of the research in this field.

Finally, the guest editors of this special issue pursue two purposes. The first is to encourage researchers acquainted with these procedures to delve further into this topic, which forms part of cutting-edge atmospheric research, so as to accelerate the process of bringing together existing analyses which, to date, are spread over a wide area. The second aim is to present a useful and suitable technique to researchers who are less experienced in this field. Including air trajectory calculation in adequate atmospheric research papers may be a useful complement that may well improve the analysis and endow it with an international scope, integrating it into a common line of research that will shed new light and bring forth fresh conclusions.

\section{Acknowledgment}

The guest editors of Advances in Meteorology hope that the papers published stimulate further developments in this useful topic.

Isidro A. Pérez

Florinda Artuso

Mastura Mahmud

Umesh Kulshrestha

M. Luisa Sánchez

M. Ángeles García 

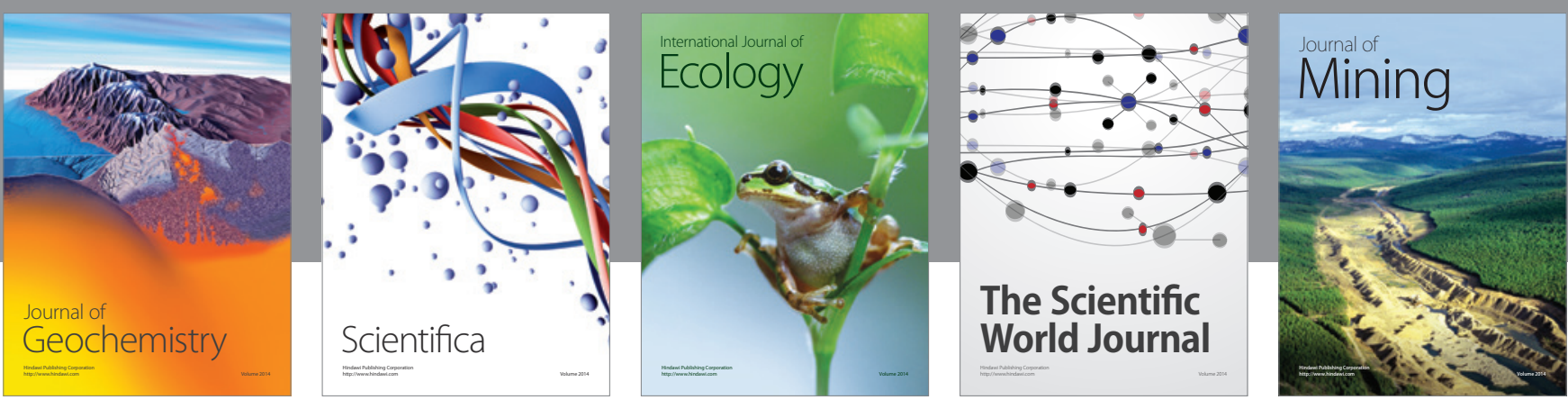

The Scientific World Journal
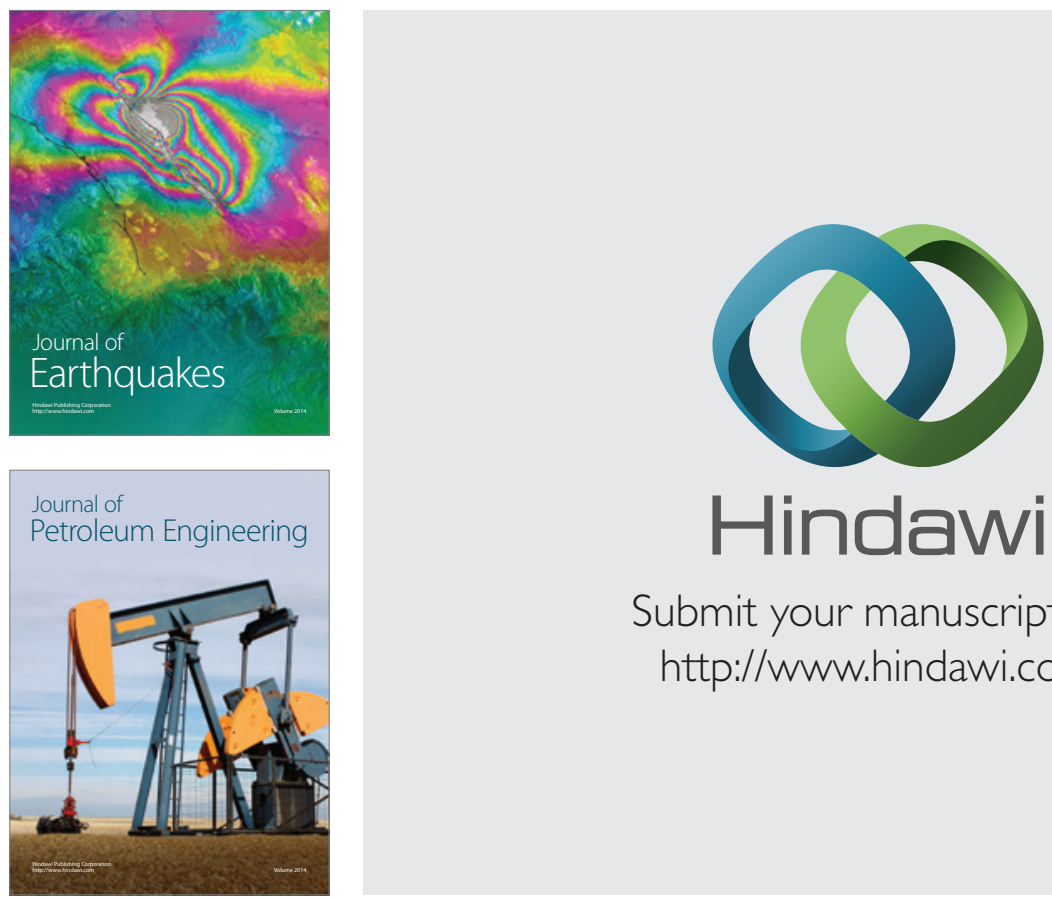

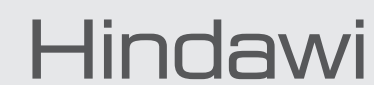

Submit your manuscripts at

http://www.hindawi.com
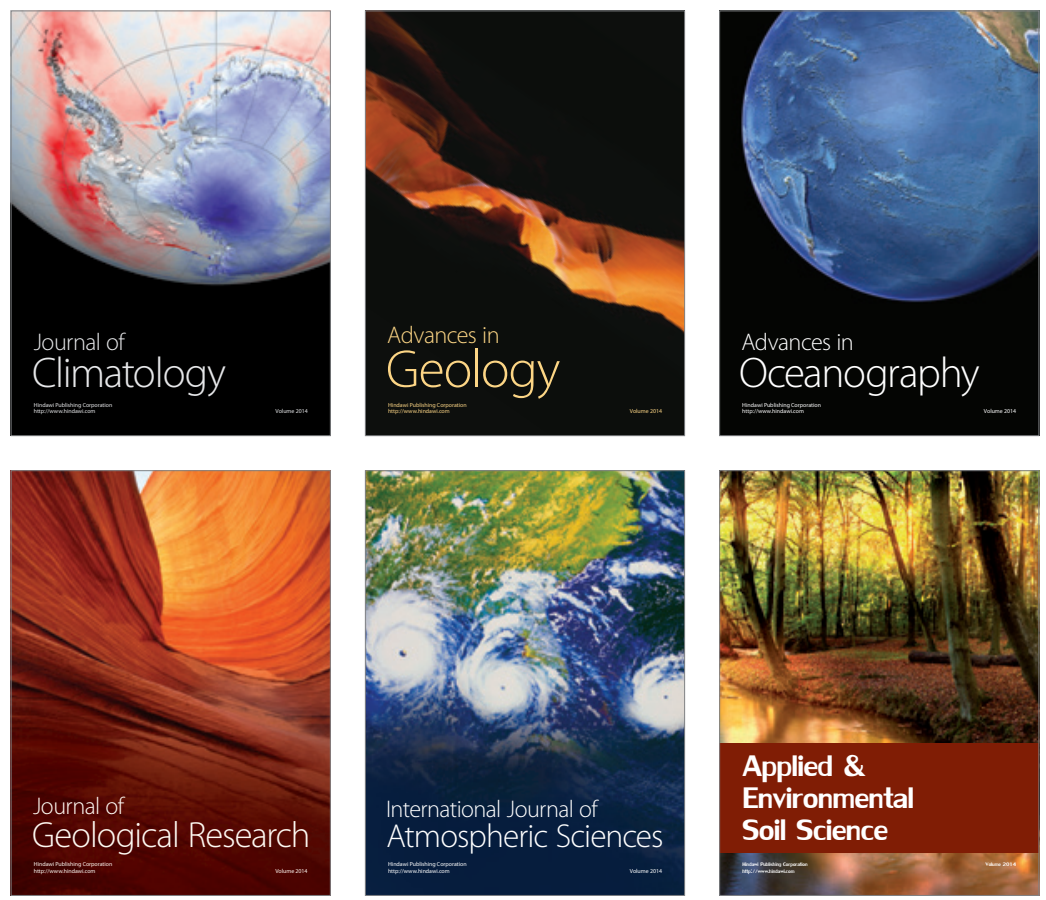
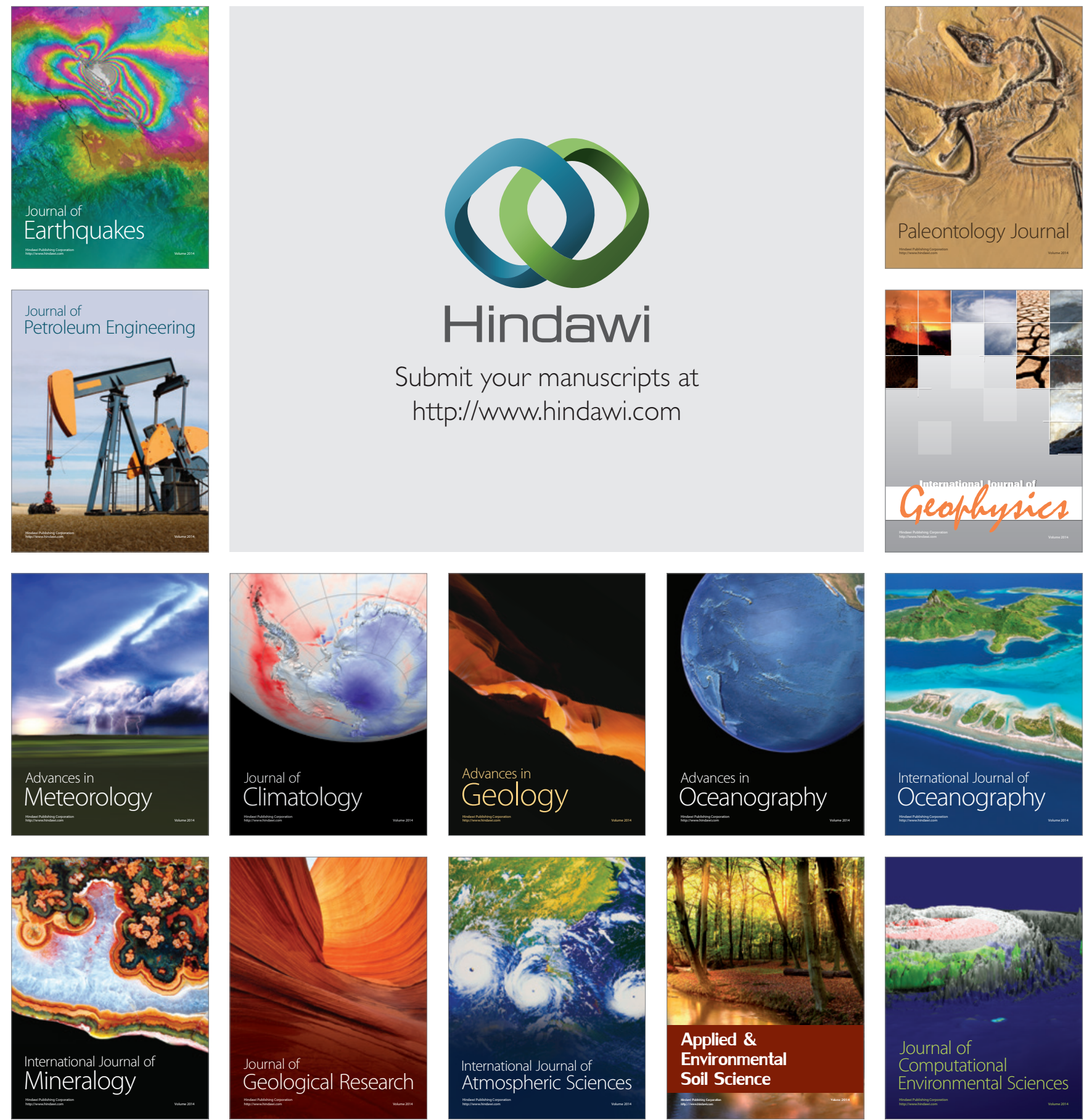